\title{
Liberalisierung und Privatisierung in Deutschland: Versuch einer Zwischenbilanz
}

Hans-Jürgen Bieling

Die politische Ökonomie der Bundesrepublik Deutschland war lange Zeit durch eine offensive Liberalisierungsstrategie, aber privatisierungspolitische Zurückhaltung gekennzeichnet. Hieran änderte sich zunächst auch wenig, nachdem die konservativ-liberale Koalition in den 1980er Jahren die Privatisierung des öffentlichen Sektors zu einem programmatischen Schwerpunkt ihrer Reformstrategie gemacht hatte. Seit den 1990er Jahren allerdings entfaltet sich eine Privatisierungsdynamik, die viele Bereiche der öffentlichen Infrastruktur erfasst. Wie die Diskussionen über die Privatisierung der Bahn und kommunaler Unternehmen verdeutlicht, ist der Prozess noch längst nicht abgeschlossen. Zugleich mehren sich aber auch die Konflikte. Die Privatisierungsskepsis in der Bevölkerung hat jüngst deutlich zugenommen.

\section{Öffentliche Unternehmen im "Modell Deutschland"}

Die Prozesse der Liberalisierung und Privatisierung sind Ausdruck einer grundlegenden Transformation des „Modell Deutschland“. Dieser Begriff, der in den 1970er Jahren als politischer Wahlslogan der Sozialdemokratie prominent geworden war, bezog sich analytisch auf einige Eigentümlichkeiten des deutschen Wirtschaftsmodells (Simonis 1998). Hierzu zählten unter anderem der hohe Stellenwert der industriellen Produktion, die koordinierende Rolle der Banken, das heißt die sogenannte "Deutschland AG“, eine Spezialisierung auf bestimmte Investitionsgüter, ein hochqualifiziertes Arbeitskräftereservoir sowie die ausgeprägte wirtschaftliche Exportorientierung, die durch eine strukturelle Unterbewertung der DMark währungspolitisch gefördert wurde. Doch nicht nur die Geldpolitik der Bundesbank, auch das System der ,ausgehandelten Demokratie“, d. h. die föderal-dezentralen Strukturen des Politikmanagements und der korporatistische Interessenausgleich, trugen mit dazu bei, dass sich die deutsche Ökonomie in den Nachkriegsjahrzehnten dynamisch entfalten konnte (Ryner 2003, S. 202ff.).

\subsection{DAS SYSTEM DER "GEMISCHTEN WIRTSCHAFTSORDNUNG"}

Es wäre jedoch verkürzt, das „Modell Deutschland" auf die Kooperation zwischen Exportindustrie und Gewerkschaften zu reduzieren. Es umschloss auch eine gemischte Wirtschaftsordnung mit einem umfassenden öffentlichen Sektor. Dessen Funktion bestand zum einen darin, betriebswirtschaftlich nicht rentable, volkswirtschaftlich aber notwendige Aufgaben zu übernehmen, um das Wirtschaftswachstum und die Arbeitsproduktivität zu fördern. Zum anderen bildete der öffentliche Sektor ein politisches Instrument, um den wirtschaftlichen Prozess aktiv zu gestalten und mit konkurrierenden sozialen Zielsetzungen in Übereinstimmung zu bringen. Hierdurch konnten nicht nur die Industriebeschäftigten, sondern auch andere soziale Gruppen an den Effekten des Wirtschaftswachstums und der Produktivitätsentwicklung als Beschäftigte und Verbraucher teilhaben, was wiederum zur Folge hatte, dass der korporatistische Klassenkompromiss auf eine relativ breite gesellschaftliche Grundlage gestellt wurde.

Im Vergleich mit anderen entwickelten kapitalistischen Gesellschaften war der öffentliche Sektor der Bundesrepublik Deutschland nach 1945 freilich durch einige Besonderheiten geprägt:

Erstens spielten staatliche Industrieunternehmen eine vergleichsweise geringe Rolle (Esser 1994, S. 108f.). In der Weimarer Republik und im Nationalsozialismus hatten sich in der Energieerzeugung, im Schiffbau und in der Automobilproduktion zwar einige kriegswirtschaftlich relevante zentralstaatliche Industriebetriebe herausgebildet (z. B. VIAG, Salzgitter AG oder VW), und auch die Länder kontrollierten - zum Teil zusammen mit dem Zentralstaat - einige Bergbau-, Elektrizitäts- und Chemieunternehmen (Tofaute 1994, S. 147ff.). Anders als in vielen anderen Staaten wurden nach dem Zweiten Weltkrieg in Westdeutschland Sozialisie- rungsforderungen jedoch nur proklamiert, nicht aber realisiert. Im Gegenteil, gegen Ende der 1950er, Anfang der 1960er Jahre kam es sogar zu einer begrenzten Teilprivatisierung staatlicher Industrieunternehmen wie Preussag, VW oder VEBA.

Angesichts des relativ bescheidenen staatlichen Industriebesitzes bestand ein zweites Merkmal des bundesdeutschen öffentlichen Sektors darin, dass er sich vornehmlich auf den Infrastrukturbereich stützte. Der Aufbau der öffentlichen Versorgungswirtschaft vollzog sich bereits während des 19. Jahrhunderts im Kontext der Industrialisierung. Nachdem zunächst die Kanalisation und Müllabfuhr sowie eine Reihe sozialer Institutionen durch die öffentliche Hand organisiert worden waren, kristallisierte sich mit der öffentlichen Wasser-, Gas- und Stromversorgung und dem öffentlichen Nahverkehr seit den 1850er Jahren der „Kern der modernen Kommunalwirtschaft heraus" (Ambrosius 2002, S. 16). Einige Infrastrukturdienstleistungen, insbesondere die großräumigen Universaldienste wie die Stromversorgung, das Post- und Fernmeldewesen oder der Eisenbahnverkehr, wurden zunehmend zentral organisiert.

Drittens war der öffentliche Infrastrukturbereich durch eine spezifische Kompetenzverteilung von Bund, Ländern und Kommunen geprägt (Esser 1994, S. 106ff.).

Hans-Jürgen Bieling, Dr., ist Juniorprofessor am Institut für Politikwissenschaft an der Philipps-Universität Marburg. Arbeitsschwerpunkte: Europäische Integration, Internationale Politische Ökonomie, Politik-, Staats- und Gesellschaftstheorie. e-mail: bieling@staff.uni-marburg.de 
Zwischen den Ebenen gab es zahlreiche Überlappungen. Der Bund konzentrierte sich jedoch vornehmlich auf die Bereitstellung großräumiger Infrastrukturdienstleistungen, wie z. B. Bahn, Post, Fernmeldewesen, Flug- und Schiffsverkehr oder Straßenbau, während die Länder für die Organisation des Bildungs- und Wissenschaftssystems, des Rundfunks oder der Landesbanken verantwortlich waren. Ein Großteil der öffentlichen Versorgungsleistungen - von der Wasser- und Energieversorgung über die Abfallbeseitigung, den ÖPNV und die Sparkassen bis hin zu Kindergärten und vielen Freizeit- und Kultureinrichtungen - lag allerdings in der Hand der Kommunen.

Die Organisation des öffentlichen Sektors veränderte sich in den Jahrzehnten nach dem Zweiten Weltkrieg nur wenig. Sie stützte sich auf einen parteiübergreifenden Konsens, dessen impliziter Referenzpunkt die Konzeption der „öffentlichen Daseinsvorsorge" war. Diese Konzeption war ursprünglich 1938 von Ernst Forsthoff im Sinne der nationalsozialistischen Gemeinschaftsideologie entwickelt, nach 1945 dann jedoch der völkischen Elemente entkleidet worden (Püttner 2000, S. 46ff.). Sie besagt im Kern, dass der Staat eine gemeinwirtschaftliche Verantwortung und aktive Rolle bei der Förderung des Gemeinwohls zu übernehmen hat. Mit der Entwicklung des „Modells Deutschland“ wurde dieser Staatsauftrag allerdings spezifisch akzentuiert. Die konjunkturpolitischen Steuerungs- und Interventionsmöglichkeiten des öffentlichen Sektors blieben angesichts des begrenzten Umfangs staatlicher Industrieunternehmen zunächst relativ bescheiden. Gegen Ende der 1960er Jahre schien sich dies mit der Konzeption der Globalsteuerung dann zu ändern. Doch faktisch wurden die - vornehmlich mittelbaren - staatlichen Industriebeteiligungen nur unwesentlich ausgeweitet; und auch die Expansion des öffentlichen Dienstleistungssektors stieß angesichts der wirtschaftlichen Stagnation und öffentlichen Verschuldung in den 1970er Jahren rasch an ihre Grenzen.

\subsection{NEOKONSERVATIVER GESELLSCHAFTSUMBAU: INKREMENTELLE PRIVATISIERUNG}

Diese Grenzen zeigten sich auch darin, dass bereits die sozial-liberale Koalition in der zweiten Hälfte der 1970er Jahre zu einer angebotsorientierten Wirtschaftspo- litik überging. Der Kurswechsel wurde durch die konservativ-liberale Regierung nach 1982 weiter forciert. Neben der Haushaltskonsolidierung ging es der neuen Regierung vor allem darum, die Marktkräfte zu stärken und den Einfluss des Staates zurückzuführen. In diesem Kontext wurde auch eine konkrete Privatisierungsstrategie entwickelt, deren Reichweite und praktische Umsetzung jedoch politisch umkämpft blieben (Mayer 2006, S. 209ff.). Eine wichtige Konfliktlinie verlief quer durch die damalige Regierungskoalition. Während der Wirtschaftsflügel der CDU und die FDP sich offensiv für die Privatisierung von Staatsunternehmen aussprachen, vertraten die Sozialausschüsse der CDU und große Teile der CSU eine eher abwehrende Position. Sie wurden dabei durch viele Bundesländer unterstützt, für die die Staatsbeteiligungen ein wichtiges industrie- und innovationspolitisches Gestaltungsinstrument darstellten. Die Spannungen innerhalb des Regierungslagers schlugen sich unter anderem darin nieder, dass die vom Finanzministerium erstellte Privatisierungsliste mit insgesamt 13 Unternehmen wiederholt modifiziert wurde. Letztlich konnte sich der Bund in der zweiten Hälfte der 1980er Jahre der Beteiligung an wichtigen Industriebetrieben -z. B.VEBA (1987), VW (1988), VIAG (1988) oder Salzgitter AG (1989) - zwar gänzlich entledigen, das Volumen der Privatisierungserlöse war mit ca. 9,4 Mrd. DM (1984-1990) jedoch gering.

In der öffentlichen Diskussion wurde die Privatisierungsstrategie der 1980er Jahre nur punktuell politisiert. Dies war zum einem dem begrenzten, weitgehend symbolischen Charakter der Privatisierung sowie der Fokussierung auf den ohnehin relativ kleinen Sektor staatlicher Industriebetriebe geschuldet (Esser 1994). Zum anderen gelang es der Sozialdemokratie und den Gewerkschaften nur vereinzelt, die negativen Beschäftigungseffekte der Privatisierung zu thematisieren, indessen makroökonomisch und demokratiepolitisch motivierte gemischtwirtschaftliche Ordnungsvorstellungen in der Öffentlichkeit kaum noch einen positiven Widerhall erzeugten (Zeuner 1998, S. 291f.). So überrascht es wenig, dass die Privatisierungsbefürworter in der Bundesregierung zunehmend offensiver agieren konnten: Erstens förderte das Bundespostministerium seit Mitte der 1980er Jahre den Übergang zu einem dualen Rundfunksystem unter Beteiligung privater Sender. Zweitens wurde
1985 eine Regierungskommission Fernmeldewesen eingerichtet, deren Überlegungen die Postreform I von 1989 anleiteten, d.h. die Aufspaltung der Bundespost in die drei selbstständigen Unternehmen Post, Telekom und Postbank. 1989 begann das Bundesverkehrsministerium drittens zudem damit, eine Konzeption für die Reform der Deutschen Bundesbahn auszuarbeiten. Viertens schließlich erhöhte die Bundesregierung seit Ende der 1980er Jahre den Druck auf die Länder und Kommunen, den zentralstaatlichen Liberalisierungs- und Privatisierungsprozess aktiv zu unterstützen.

\section{Erweiterung der Liberalisierungs- und Privatisierungsagenda}

In den 1990er Jahren erfasste die Privatisierungsstrategie auch den Bereich der öffentlichen Daseinsvorsorge. Hierbei handelte es sich um einen Bereich, dessen staatliche Organisation politisch-kulturell verankert war. Eigentlich wäre daher eine wiederholte Blockade der Liberalisierungs- und Privatisierungsinitiativen zu erwarten gewesen. Doch genau diese Blockade fand nicht statt. Im Gegenteil, nach anfänglichem Zögern konnte der Paradigmenwechsel hin zu einer marktliberalen Reorganisation des öffentlichen Infrastrukturbereichs relativ umfassend durchgesetzt werden. Der tradierte „Produktions-“ oder „Leistungsstaat", der für die Bereitstellung der öffentlichen Infrastruktur selbst verantwortlich ist, wurde Schritt für Schritt demontiert und durch einen "Regulierungs-“ oder "Gewährleistungsstaat" ersetzt, der sich darauf beschränkt, die privatwirtschaftliche Organisation und das Angebot öffentlicher Versorgungsleistungen zu kontrollieren (Grande/Eberlein 2000). Dieser Prozess wurde dadurch gefördert, dass sich in der Folge der deutschen Einheit und europäischen Integration die politischen Diskurse und Entscheidungsparameter verschoben und sich auch die Handlungsspielräume für alternative Organisationsformen verengt hatten.

\subsection{DIE DEUTSCHE EINHEIT UND DIE FOLGEN}

Im Kontext der deutschen Einheit vollzog sich zunächst ein gewisser Bruch mit der 
bisherigen - verhandlungspolitisch gebremsten - Privatisierungsstrategie. Da der öffentliche Sektor nach der Wende von 1989 vorübergehend stark angewachsen war, wurde die Privatisierung der Volkseigenen Betriebe (VEBs) der Treuhandanstalt übertragen, einer eigenständigen, dem Finanzministerium unterstellten Institution des öffentlichen Rechts (Grosser 2003). Schon die Volkskammer der DDR hatte im März 1990 den Beschluss gefasst, diese von der Regierung kontrollierte Industrie-Holding zu errichten, um die bestehenden Kombinate zu entflechten und in marktfähige Kapitalgesellschaften umzuwandeln. Durch das Treuhandgesetz vom Juni 1990, das die Regierung de Maizière in enger Kooperation mit der bundesdeutschen Regierungskoalition aus CDU, CSU und FDP entworfen hatte, wurde der Privatisierungs- und Reorganisationsauftrag weiter präzisiert; und zwar dahingehend, dass die Privatisierung Vorrang haben sollte gegenüber sanierungs- und industriepolitischen Erwägungen (Mayer 2006, S. 222).

Der Treuhand waren anfangs etwa 8.500 VEBs - durch die Entflechtung stieg die Zahl später noch deutlich an - mit etwa 4,1 Mio. Beschäftigten unterstellt. Die Privatisierung und Sanierung der VEBs erwies sich als sehr schwierig, weil viele der Betriebe bereits zu DDR-Zeiten unrentabel waren. Durch die Währungsunion und die Konkurrenz mit westdeutschen und europäischen Unternehmen wurde die Situation weiter verschärft. Der Treuhand gelang es bis zu ihrer Auflösung Ende 1994 zwar, knapp 8.000 Betriebe vollständig zu privatisieren oder zu kommunalisieren, zugleich mehrten sich jedoch die wirtschaftlichen und sozialen Kosten der privatisierungspolitisch dominierten Reorganisationsstrategie. So wurden über 3.700 Unternehmen liquidiert; die Zahl der Beschäftigten in den ehemaligen VEBs schrumpfte von 4,1 auf 1,5 Mio. Personen; und das ursprünglich proklamierte Ziel, die Kosten der deutschen Einheit durch die Privatisierung finanzieren zu können, wurde weit verfehlt (Grosser 2003). Angesichts der Ausgaben für Sozialpläne, laufende Betriebsverluste, Bankenbürgschaften und andere Investitions- und Subventionszusagen beendete die Treuhandanstalt ihre Arbeit letztlich mit einer Gesamtverschuldung von über 250 Mrd. DM, die nach 1994 auf mehrere bundesstaatliche Folgegesellschaften aufgeteilt wurde (Rügemer 2006, S. 39f.).
Trotz dieses Verlustgeschäftes erhöhte sich in der Folge der deutschen Einheit der Privatisierungsdruck. Dies lag vor allem daran, dass sich die Reproduktionsbedingungen des „Modells Deutschland“ beträchtlich gewandelt hatten. Auf der einen Seite wurde die Exportorientierung der deutschen Unternehmen nochmals gestärkt. Bereits der Aufbau Ost war ein riesiges Konjunkturprogramm für die westdeutsche Industrie. Zudem sorgten die arbeitsmarkt- und sozialpolitische Deregulierung, die Lohnzurückhaltung der Gewerkschaften und die steuerliche Entlastung der Unternehmen dafür, dass die deutsche Ökonomie zuletzt wieder einen beträchtlichen Leistungsbilanzüberschuss verzeichnen konnte (Huffschmid 2007, S. 314f.). Auf der anderen Seite waren und sind die finanziellen Belastungen der deutschen Einheit aber enorm. Der Nettofinanztransfer von West nach Ost belief sich für die Zeit von 1991 bis 2003 auf insgesamt 980 Mrd. $€$ (Busch 2005, S. 82). Die Aufbringung dieser Summe - durch erhöhte Sozialversicherungsbeiträge und indirekte Steuern - belastete nicht nur die Beschäftigten und Konsumenten, sondern auch die öffentlichen Haushalte, deren Verschuldung sich von $536 \mathrm{Mrd}$. $€(1990)$ auf $1.566 \mathrm{Mrd}$. $€$ (2006) erhöhte (Bajohr 2007, S. 209).

\subsection{EUROPÄISCHE INTEGRATION UND REORGANISATION ÖFFENTLICHER DASEINSVORSORGE}

Neben der deutschen Einheit stimulierte auch der Prozess der europäischen Integration die Privatisierungspolitik der 1990er Jahre. Von besonderer Bedeutung waren einige sektorale Liberalisierungsrichtlinien, die sich auf zentrale Bereiche der öffentlichen Infrastruktur und Daseinsvorsorge bezogen: so vor allem auf den Telekommunikationssektor, das Postwesen, den Luftverkehr, die Bahn sowie die Strom- und Gasversorgung. In all diesen Bereichen kristallisiert sich seit Ende der 1980er Jahre ein neues europäisches Regulierungsmodell heraus (Lippert 2005, S. 19ff.; Bieling/Deckwirth 2008). Dieses Modell zielt darauf, die ehemaligen staatlichen Monopolunternehmen zu entflechten, d.h. die öffentlich garantierten Infrastrukturnetze von deren Nutzung durch Dienstleistungsanbieter zu trennen, um den grenzüberschreitenden Wettbewerb zu fördern. Das europäische Regulierungsmodell schreibt keineswegs eine materielle Privatisierung der öffentlichen Infrastrukturunternehmen vor. Es definiert durch die wettbewerbsrechtlichen Elemente der Liberalisierungsrichtlinien jedoch einen Handlungsrahmen, der staatliche Beihilfen und Subventionen erschwert und eine formelle Privatisierung, d.h. die Umwandlung von öffentlich-rechtlichen in privatrechtlich organisierte Unternehmen, begünstigt. Genau diese Vorgaben wurden vonseiten der konservativ-liberalen Regierungskoalition in den 1990er Jahren bereitwillig aufgegriffen: erstens, um die ordnungspolitische Entstaatlichungsstrategie zu beschleunigen; zweitens, um die öffentlichen Haushalte durch Privatisierungserlöse wenigstens partiell zu entlasten; und drittens, um durch die Mobilisierung privaten Kapitals die infrastrukturpolitischen Investitionslücken zu schließen, die sich durch den Vereinigungsprozess noch vergrößert hatten.

\section{POST- UND TELEKOMMUNIKATIONS- SEKTOR}

Im Zentrum der Privatisierungspolitik stand zunächst der Post- und Telekommunikationssektor (Deckwirth 2008). Der Prozess war bereits durch die Postreform I von 1989, d.h. durch die Aufspaltung der Bundespost in die Unternehmen Post, Telekom und Postbank eingeleitet worden. Vor dem Hintergrund der Finanzprobleme, die durch die Modernisierung der Infrastruktur in Ostdeutschland entstanden waren, und der Liberalisierungserfordernisse des EG-Binnenmarktes verständigte sich die Bundesregierung mit der SPD gegen den Widerstand der Deutschen Postgewerkschaft (DPG) - auf die Postreform II, die Anfang 1995 in Kraft trat (Zohlnhöfer 2001, S. 340ff.). Die Abstimmung mit der SPD erfolgte mit Blick auf eine notwendige Änderung des Art. 87 des Grundgesetzes, durch die das leistungsstaatliche Konzept des Verwaltungsmonopols durch die Grundsätze einer privatwirtschaftlichwettbewerbsorientierten Infrastrukturpolitik ersetzt wurde. ${ }^{1}$ Nur so konnten die

\footnotetext{
Nach der Grundgesetzänderung vom Dezember 1993 waren auf Bundesebene nur noch wenige Bereiche von einer Privatisierung verfassungsrechtlich ausgeschlossen: „der Auswärtige Dienst, die Bundesfinanzverwaltung, die Verwaltung der Bundeswasserstraßen und der Schifffahrt, die Bundeswehr(verwaltung), die Luft- und Eisenbahnverkehrsverwaltung, die Bundesbank sowie die rechtsprechende Gewalt" (Bajohr 2007, S. 240).
} 
drei Unternehmen Post, Telekom und Postbank in eigenständige Aktiengesellschaften umgewandelt, also formell, später dann auch materiell, privatisiert werden. Im Fall der Deutschen Telekom erfolgte die materielle Privatisierung sehr schnell. Schon 1996 wurde das Unternehmen im Anschluss an eine Werbekampagne für „Volksaktien" an der Börse platziert, bevor die rot-grüne Koalition die materielle Privatisierung durch weitere Aktienverkaufsprogramme in den Jahren 1999 und 2000 fortsetzte. Ende 2006 verfügte der Bund direkt nur noch über $16,9 \%$ und indirekt, d.h. über die Kreditanstalt für Wiederaufbau (KfW), nur noch über 14,8 \% der Unternehmensanteile. Im Vergleich zur Telekom lief die Privatisierung der Deutschen Post etwas später an. Im Anschluss an den Börsengang im Jahr 2000 beschleunigte sich der Prozess jedoch enorm. 2005 hatte der Bund seine Mehrheitsbeteiligung aufgegeben, sodass die KfW inzwischen nur noch über einen Anteil von 30,5 \% verfügt (Bundesministerium der Finanzen 2007, S. 7f.).

Nachdem die Postreform II die Privatisierung ermöglicht hatte, folgten weitere Schritte, um den deutschen Markt in Übereinstimmung mit den europäischen Richtlinien zu liberalisieren. In beiden Sektoren wurde 1998 die Wettbewerbs- und Aufgabenregulierung aus dem Postministerium ausgelagert und der autonomen Regulierungsbehörde für Telekommunikation und Post (RegTP), seit 2005 der Bundesnetzagentur (BNetzA) übertragen, die darüber hinaus auch für die Regulierung der Stromund Gasmärkte sowie des Eisenbahnverkehrs zuständig ist. Die Bundesnetzagentur konnte den Liberalisierungsprozess zwar verstetigen und die Marktdominanz der ehemaligen Monopolunternehmen aufweichen, nicht aber durchbrechen. Mehr noch, sowohl die Deutsche Telekom als auch die Deutsche Post haben die europäische und zum Teil auch globale Liberalisierung der Märkte aktiv genutzt, um sich selbst zu internationalisieren (Schröter 2007, S. 83ff.; Deckwirth 2008). Die Deutsche Post hat sich durch den Kauf von Logistikunternehmen wie DHL, Danzas oder Exel zu einem Global Player entwickelt. Von den insgesamt 536.000 Mitarbeitern sind inzwischen nur noch ca. $40 \%$ in Deutschland beschäftigt (Deutsche Post World Net 2007, S. 71). Ähnliches gilt für die Deutsche Telekom; auch sie erzielt mittlerweile über die Hälfte der Unternehmenserlöse im Ausland (Deutsche Telekom 2007).

\section{BAHNVERKEHR}

Im Vergleich zum Post- und Telekommunikationssektor stellt sich der Liberalisierungs- und Privatisierungsprozess im Bahnverkehr komplizierter dar. Dies liegt vor allem daran, dass durch die Konkurrenz des Straßen- und Luftverkehrs die Deutsche Bundesbahn (DB) bereits in den 1980er Jahren finanziell unter Druck geraten war und sich die Finanzprobleme im Zuge der deutschen Einheit, d.h. durch den Einbruch des Gütertransports für die Deutsche Reichsbahn (DR) und einen großen Modernisierungs- und Investitionsbedarf in Ostdeutschland, weiter zuspitzten. Die erste Stufe der Bahnreform, eingeleitet mit Inkrafttreten des Eisenbahnneuordnungsgesetzes Anfang 1994, umfasste dabei folgende Elemente: erstens die Entschuldung durch den Bund sowie die Zusammenführung der DB und DR zur Deutschen Bahn AG, d.h. die formelle Privatisierung durch die Umwandlung in eine Kapitalgesellschaft; zweitens die Trennung von Schienennetz und Fahrbetrieb, die Einrichtung des Eisenbahnbundesamtes (EBA) als Regulierungsbehörde sowie die grundsätzliche Marktöffnung für andere Wettbewerber gemäß den europäischen Liberalisierungsvorgaben; und drittens eine Regionalisierung des schienengebundenen Personennahverkehrs, durch die die Deutsche Bahn AG entlastet wurde und gemeinwirtschaftliche Aufgaben den Bundesländern übertragen wurden.

Die Vorstandsvorsitzenden, von Heinz Dürr über Johannes Ludewig bis Hartmut Mehdorn, waren seit den 1990er Jahren darauf bedacht, die Deutsche Bahn AG zu einem modernen, international wettbewerbsfähigen Konzern zu entwickeln. Neben den Finanzierungs- und Investitionszusagen des Bundes sollte dies durch die Stilllegung unrentabler Streckenabschnitte, einen umfassenden Personalabbau, die Akquisition global operierender Logistikunternehmen wie Schenker/Railion und BAX Global und eine Unternehmensreorganisation geschehen. Mit der zweiten Stufe der Bahnreform von 1999 wurde die Deutsche Bahn AG in eine Holding mit fünf eigenständigen Tochterunternehmen umgewandelt. Ungeachtet dieser Maßnahmen konnten die angestrebten Ziele jedoch nur bedingt realisiert werden (Deckwirth 2008): Nach der Entschuldung häufte die Deutsche Bahn AG von 1994 bis 2004 erneut Verbindlichkeiten von über 38 Mrd. $€$ an (Engartner 2005, S. 916); und trotz der Liberalisierung konnten sich bislang nur wenige private Anbieter im Personenverkehr - etwas stärker im Güterverkehr etablieren. Der angestrebte Börsengang läuft nach kontroversen öffentlichen Diskussionen über die Perspektiven des Service-Angebots nur auf eine Minimallösung hinaus. Die geplante Holding für den Fahrbetrieb und die Logistik soll nur zu maximal $24,9 \%$ privatisiert werden, während das Schienennetz gänzlich im Besitz des Bundes bleibt.

\section{ENERGIEVERSORGUNG}

Ein weiterer Bereich, in dem europäische Liberalisierungsvorgaben die Privatisierung begünstigt haben, ist die Energieversorgung. Die großen privatrechtlich organisierten Verbundunternehmen dominierten zwar schon länger den deutschen Energiemarkt. Ihr Operationsfeld beschränkte sich jedoch auf den Kraftwerkbetrieb und die großen Leitungsnetze, während der Endverbrauch den dezentralen Kommunalunternehmen, d.h. vor allem den Stadtwerken, vorbehalten war (Baur 2002). Wie in den anderen Infrastruktursektoren hatte die EU 1996 eine Trennung von Netzbetrieb und Netznutzung und einen liberalisierten Netzzugang für konkurrierende Unternehmen vorgeschrieben. In Deutschland wurde diese Vorgabe mit dem neuen Energiebewirtschaftungsgesetz von 1998 umgesetzt. Zugleich änderte sich hierdurch die bis dahin bestehende Versorgungsstruktur. Einerseits wurden die Energieunternehmen durch die Liberalisierung - zumindest potenziell - einem größeren Wettbewerb ausgesetzt. Andererseits eröffnete die Aufhebung der lokalen Monopolstrukturen den Versorgungsunternehmen die Möglichkeit, auch den Endverbrauch zu übernehmen.

Angesichts der schwachen wettbewerbspolitischen Kontrollmechanismen in Deutschland wurde zunächst nur das Modell eines zwischen den Markteilnehmern ausgehandelten Netzzugangs etabliert - wurde durch die Liberalisierung die Fusionswelle in der Strom- und Gasversorgung nochmals verstärkt. Im Prinzip ist der Strommarkt mittlerweile zwischen vier großen Verbundunternehmen - RWE, E.ON, EnBW und Vattenfall Europe aufgeteilt. Über die Tochter-Unternehmen E.ON Ruhrgas und RWE-Thyssengas kontrollieren E.ON und RWE auch den 


\begin{tabular}{|c|c|c|c|c|c|}
\hline Jahr & $\begin{array}{l}\text { Transaktions- } \\
\text { volumen } \\
\text { (in US \$) }\end{array}$ & $\begin{array}{l}\text { Kapitalmarkt- } \\
\text { veräußerungen } \\
\text { (in US \$) }\end{array}$ & $\begin{array}{c}\text { Zahl der } \\
\text { Kapitalmarkt- } \\
\text { transaktionen }\end{array}$ & $\begin{array}{c}\text { Zahl der unmittelbaren } \\
\text { Bundesbeteiligungen und } \\
\text { Sondervermögen des Bundes }\end{array}$ & $\begin{array}{c}\text { Zahl der Bundesbeteiligungen } \\
\text { mit einem Nennwert von mind. } \\
25 \% \text { und } 50.000 €\end{array}$ \\
\hline 1987 & 275,60 & 275,60 & 1 & - & - \\
\hline 1988 & 816,60 & 816,60 & 3 & - & - \\
\hline 1989 & $1.751,00$ & 682,60 & 2 & - & - \\
\hline 1990 & 306,87 & - & 0 & - & - \\
\hline 1991 & $2.877,48$ & 240,70 & 1 & 214 & 136 \\
\hline 1992 & 565,09 & - & 0 & - & - \\
\hline 1993 & 563,62 & - & 0 & 209 & 107 \\
\hline 1994 & $4.906,99$ & $2.140,00$ & 2 & - & - \\
\hline 1995 & $1.302,98$ & - & 0 & 144 & 73 \\
\hline 1996 & $13.993,48$ & $12.487,00$ & 1 & 143 & 74 \\
\hline 1997 & $6.567,40$ & $2.480,50$ & 1 & 144 & 74 \\
\hline 1998 & $3.282,69$ & 814,30 & 2 & 137 & 70 \\
\hline 1999 & $13.356,78$ & $10.365,50$ & 2 & 131 & 66 \\
\hline 2000 & $19.373,29$ & $17.391,80$ & 2 & 128 & 64 \\
\hline 2001 & $5.074,44$ & $1.160,00$ & 2 & 122 & 60 \\
\hline 2002 & 420,98 & - & 0 & 120 & 37 \\
\hline 2003 & $1.322,52$ & $1.221,40$ & 1 & 116 & 36 \\
\hline 2004 & $15.440,70$ & $6.321,48$ & 2 & 111 & 32 \\
\hline 2005 & $3.632,29$ & $3.240,73$ & 2 & 108 & 33 \\
\hline 2006 & $10.546,74$ & $1.652,20$ & 1 & 112 & 33 \\
\hline 2007 & $9.163,06$ & $3.258,50$ & 2 & 110 & 33 \\
\hline Gesamt & $115.540,58$ & $64.548,91$ & 27 & & \\
\hline
\end{tabular}

deutschen Gasmarkt, wobei der Gastransport zugleich durch einige andere Unternehmen wie die BEB oder Wingas, ein Gemeinschaftsunternehmen von Wintershall (BASF) und Gazprom, mit organisiert wird. In den letzten Jahren haben die deutschen Energiekonzerne nicht nur in vielen europäischen Ländern Marktanteile erobert (Schröter 2007, S. 79ff.), sondern auch innerhalb Deutschlands durch die Übernahme städtischer Versorgungsbetriebe ihre marktbeherrschende Position ausgebaut.

\section{PRIVATISIERUNGSERLÖSE UND -KOSTEN}

Für die öffentlichen Haushalte stellt sich die Privatisierung auf den ersten Blick recht positiv dar. Im Laufe der 1990er Jahre gelang es dem Bund nicht nur, die Zahl der mittelbaren und unmittelbaren Beteiligungen zu reduzieren (Bundesministerium der Finanzen 2007), sondern er konnte zwischen 1996 und 2007 auch beträchtliche Privatisierungserlöse im Umfang von etwa $100 \mathrm{Mrd}$. US \$ realisieren (Tabelle 1).

Diesen Einnahmen stehen auf der anderen Seite aber auch erhebliche Kosten gegenüber. Denn bevor die ehemals staatlichen Unternehmen an der Börse platziert werden konnten, mussten sie zum Teil durch öffentliche Unterstützungsleistungen - z. B. durch Entschuldungsprogramme, Investitionszusagen, steuerliche Vergünstigungen oder erhöhte Gebühren rentabel und wettbewerbsfähig gemacht werden (Rügemer 2006, S. 67ff.). Diese Sanierungs- und Subventionskosten lassen sich nur schwer quantifizieren. Sie haben jedoch mit dazu beigetragen, die Position der deutschen Infrastrukturkonzerne in der internationalen Konkurrenz zu stärken.

\subsection{FINANZKRISE ÖFFENTLICHER HAUSHALTE: FOLGEN FÜR DIE KOMMUNALWIRTSCHAFT}

Während der Liberalisierungs- und Privatisierungsprozess durch das Zusammenspiel von Bundesregierung, Europäischer Kommission und privaten Dienstleistungsanbietern vorangetrieben wurde, verhielten sich die Bundesländer und Kommunen - zumindest in Bezug auf die materielle Privatisierung - zunächst zögerlicher (Kamm 2003, S. 135ff.). Viele Länderregierungen verbanden mit ihrem Besitz oder ihren Beteiligungen an Kredit-, Verkehrsund Energieunternehmen oder Wohnungsbaugesellschaften immer auch die Option einer aktiven Industrie-, Struktur- und Sozialpolitik. Gleiches gilt im Prinzip auch für die Kommunen, deren Besitz- und Beteiligungsspektrum sehr umfassend ist: Neben den klassischen Feldern der Kommunalwirtschaft - wie Energie- und Wasserversorgung, Abwasser und Müllabfuhr, Öffentlicher Personennahverkehr (ÖPNV) und Sparkassen - zählen hierzu auch die Aufgaben der Gebäudebewirtschaftung, des Kultur- und Freizeitangebots (Büchereien, Schwimmbäder etc.) und eine Reihe sozialer Dienste wie Rettungsdienste, Krankenhäuser, Kindergärten oder Altenheime, die zum Teil allerdings in Kooperation mit freien Trägern ausgefüllt werden (Bremeier et al. 2006, S. 31).

Im Laufe der 1990er Jahre gaben viele Länder und Kommunen ihre zum Teil privatisierungsskeptische Grundhaltung zunehmend auf und sahen in der Ausgliederung öffentlicher Aufgaben eine Option, ihre Haushalte zu entlasten und den steigenden Investitionsbedarf zu decken. Öffentliche, in Eigenregie geführte Betriebe wurden vermehrt in privatrechtliche Unternehmen umgewandelt und nach betriebswirtschaftlichen Gesichtspunkten reorganisiert. Dies betraf vor allem die Stadtwerke, den ÖPNV oder die Wohnungswirtschaft. Nicht selten handelte es sich bei der eigentumsrechtlichen Privatisierung nur um einen ersten Schritt, dem schon bald eine materielle Privatisierung folgte, 
die sich über die Veräußerung des Immobilienbestandes hinaus insbesondere auf den Verkauf der Versorgungsbetriebe, der Schwimmbäder und Krankenhäuser konzentrierte (Hauschild et al. 2004, S. 56f.). In den letzten Jahren mehren sich zudem Public Private Partnerships, d.h. Projekte einer funktionalen und zeitlich begrenzten Ausgliederung spezifischer Teilaufgaben. Manche Gemeinden übertragen für etwa 15 bis 30 Jahre den Bau, die Sanierung oder die Organisation und den Betrieb öffentlicher Einrichtungen an Privatunternehmen, um im Gegenzug ihrerseits eine fortlaufende Gebühr oder Miete zu entrichten (Deutsches Institut für Urbanistik 2005; Rügemer 2006, S. 93ff.).

In der Folge all dieser Maßnahmen konnten sich die Kommunen finanziell entlasten und zwischen 2000 und 2006 Vermögensveräußerungen von durchschnittlich etwa $6,5 \mathrm{Mrd}$. $€$ pro Jahr realisieren (Janetschek 2007, S. 11). Der Verschuldungsanstieg wurde hierdurch abgebremst. Zugleich sind jedoch die öffentlichen Sachinvestitionen in den letzten beiden Jahrzehnten stetig zurückgegangen. Weite Bereiche der kommunalen Daseinsvorsorge sind inzwischen sehr stark von privaten Investitionen abhängig, die ihrerseits nur mit Aussicht auf ansprechende Gewinne getätigt werden. Diese ökonomische Abhängigkeit korrespondiert mit einem zunehmenden politischen Kontrollverlust (Sack 2006). Dieser manifestiert sich zum Teil bereits im finanzpolitisch bedingten Veräußerungs- oder Auslagerungsprozess, um sich danach zu verstetigen. So kristallisieren sich auch auf der Länder- und Kommunalebene Elemente eines exekutivdominierten Regulierungs- und Gewährleistungsstaates heraus, dessen Operationsweise von den parlamentarischen Institutionen nur noch begrenzt überwacht und beeinflusst werden kann.

\section{Perspektiven der Privatisierung}

Die Prozesse der Liberalisierung und Privatisierung haben sich in Deutschland seit
Mitte der 1990er Jahre beschleunigt. Nach dem Platzen der Aktienblase in den Jahren 2001 und 2002 wurden kapitalmarktvermittelte Privatisierungsschritte zwar vorübergehend gebremst, um anschließend wieder Fahrt aufzunehmen. Vor allem auf der Länder- und Kommunalebene gibt es neben den Versorgungsbetrieben und dem Gebäudemanagement noch viele Bereiche der sozialen Infrastruktur - Forschungsund Bildungseinrichtungen, Kindergärten oder Krankenhäuser -, die perspektivisch privatisiert oder ausgelagert werden können; und bundesweit schreitet die Teilprivatisierung der sozialen Sicherungssysteme, insbesondere in den Bereichen der Alterssicherung („Riester-Rente“) und Gesundheit, weiter voran. Gemäß dem ordnungspolitischen Leitbild eines modernen und wirtschaftlich effizienten öffentlichen Sektors sollen immer mehr Leistungen unter staatlicher Aufsicht nur noch von Privatunternehmen erbracht werden. Der Paradigmenwechsel vom Leistungs- zum Gewährleistungsstaat verweist dabei auf veränderte politische Organisationsnetzwerke. Die Prinzipien und die Funktionsweise der öffentlichen Daseinsvorsorge werden kaum mehr durch die alten fordistischen Netzwerke aus Regierungsvertretern, Verwaltungsfachleuten, parlamentarischen Kontrollgremien und Gewerkschaftsrepräsentanten bestimmt, sondern zunehmend durch neue Allianzen aus marktliberalen Politikern, ConsultingAgenturen, Finanzmarktakteuren (Shareholdern) und privatwirtschaftlich geschulten Managern.

Im Zuge der Liberalisierung und Privatisierung transformiert sich daher auch das institutionelle Gefüge, durch das das Modell Deutschland lange abgestützt wurde (Beck/Scherrer 2005, S. 116f.). Die tradierten Formen des föderalen und korporatistischen Politikmanagements werden nicht einfach hinfällig, aber nach Maßgabe modernisierungs- und wettbewerbspolitischer Zielsetzungen neu justiert. So ist der politische Reformprozess durch einen schleichenden Übergang von einem „kooperativen“ zum „kompetitiven“ Föderalismus und eine Erosion gewerkschaftlicher Mitsprache- und Einflussmöglichkeiten geprägt. Die Elemente eines solidarischen - regionalen und sozialen - Interessenausgleichs werden hierdurch offenkundig geschwächt. In vielen der privatisierten Unternehmen sind ein beträchtlicher Arbeitsplatzabbau und eine vielfach verschlechterte Qualität der Beschäftigungsverhältnisse zu beobachten; und auch die Qualität der Versorgungsdienstleistungen hat - in der Folge steigender Nutzungskosten und einer ausgedünnten Flächenpräsenz - in vielen Bereichen abgenommen (Hauschild et al. 2004, S. 43ff.).

Angesichts dieser Tendenzen kam im Januar 2008 eine Umfrage, die das Meinungsforschungsinstitut Forsa im Auftrag des Deutschen Beamtenbundes durchgeführt hatte, zu dem Ergebnis, dass die Privatisierungsskepsis in der deutschen Bevölkerung deutlich zugenommen hat. Eine Preis- und Qualitätsverbesserung sehen die meisten allein bei Telekommunikationsdienstleistungen, während in vielen anderen Bereichen wie z. B. der Post, der Energieversorgung und dem Bahnverkehr steigende Preise und - mit Ausnahme des Paketdienstes und der Müllversorgung - auch eine beeinträchtigte Dienstleistungsqualität beobachtet werden. Doch nicht nur dieser Stimmungsumschwung, auch die wachsende Zahl von Volks- und Bürgerbegehren, die sich auf der Landes- oder Kommunalebene gegen geplante Privatisierungsvorhaben richten, signalisiert, dass die Organisation und Bereitstellung von Infrastruktur- und Versorgungsdienstleistungen in den letzten Jahren vermehrt politisiert wird (Mittendorf 2008). Der Privatisierungsprozess stellt demzufolge nicht notgedrungen eine Einbahnstraße dar. Angesichts des politischen Kontrollverlustes und unerfüllter Effizienzerwartungen werden zudem in etwa $10 \%$ der deutschen Kommunen Überlegungen angestellt, privatisierte Betriebe wieder zu rekommunalisieren (Janetschek 2007, S. 18). Noch handelt es sich hierbei zumeist nur um vage Absichtsbekundungen. Diskurse über eine alternative gemeinwirtschaftliche Organisation der öffentlichen Daseinsvorsorge entwickeln sich allenfalls ansatzweise. $\mathrm{Ob}$ der laufende Privatisierungsprozess perspektivisch aufgehalten oder sogar umgekehrt werden kann, bleibt daher ungewiss. 
Ambrosius, G. (2002): Die historische Entwicklung der öffentlichen Daseinsvorsorge in Deutschland unter aktueller europäischer Perspektive, in: Hrbek, R./Nettesheim, M. (Hrsg.): Europäische Union und mitgliedstaatliche Daseinsvorsorge, Baden-Baden, S. 15-31

Bajohr, S. (2007): Grundriss Staatliche Finanzpolitik. Eine praktische Einführung, Wiesbaden

Baur, J. (2002): Energieversorgung durch Stadtwerke, in: Hrbek, R./Nettesheim (Hrsg.): Europäische Union und mitgliedstaatliche Daseinsvorsorge, Baden-Baden, S. 118-122

Beck, S./Scherrer, C. (2005): Der rot-grüne Einstieg in den Abschied vom "Modell Deutschland“ : Ein Erklärungsversuch, in: Prokla 1, S. 111-130 Bieling, H.-J./Deckwirth, C. (2008): Die Reorganisation der öffentlichen Infrastruktur in der Europäischen Union - Einleitung, in: Bieling, H.-J./ Deckwirth, C./Schmalz, S. (Hrsg.): Liberalisierung und Privatisierung in Europa. Die Reorganisation der öffentlichen Infrastruktur in der Europäischen Union, Münster, S. 9-33

Bremeier, W./Brinckmann, H./Killian, W. (2006): Kommunale Unternehmen in kleinen und mittelgroßen Kommunen sowie Landkreisen, in: Killian, W./Richter, P./Trapp, J. H. (Hrsg.): Ausgliederung und Privatisierung in Kommunen, Berlin

Bundesministerium der Finanzen (BMF) (2007): Beteiligungsbericht 2007, Berlin

Busch, U. (2005): Aufbau Ost - Bilanz und Perspektiven, in: Berliner Debatte Initial 1, S. 79-90

Deckwirth, C. (2008): Der Erfolg der Global Player. Liberalisierung und Privatisierung der öffentlichen Infrastrukturdienstleistungen in der Bundesrepublik Deutschland, in: Bieling, H.-J./Deckwirth, C./Schmalz, S. (Hrsg.): Liberalisierung und Privatisierung in Europa. Die Reorganisation der öffentlichen Infrastruktur in der Europäischen Union, Münster,

S. 64-95

Deutsche Post World Net (2007): Roadmap to Value. Geschäftsbericht 2007, http://investors.dpwn.de/de/investoren/publikationen/archiv/ 2007/finanzpublikationen/dpwn_annual_report_2007_de.pdf Deutsche Telekom (2007): Connected life and work. Das Geschäftsjahr 2007, www.download-telekom.de/dt/StaticPage/50/77/04/080228_ dtag_gb_2007.pdf_507704.pdf

Deutsches Institut für Urbanistik (2005): Public Private Partnership Projekte. Eine aktuelle Bestandsaufnahme in Bund, Ländern und Gemeinden, Berlin

Engartner, T. (2005): Zug nach Nirgendwo, in: Blätter für deutsche und internationale Politik 8, S. 916-918

Esser. J. (1994): Germany: Symbolic Privatizations in a Social Market Economy, in: Wright, V. (Hrsg.): Privatization in Western Europe: Pressures, Problems and Paradoxes, London, S. 105-121

Grande, E./Eberlein, B. (2000): Der Aufstieg des Regulierungsstaates im Infrastrukturbereich. Zur Transformation der politischen Ökonomie der Bundesrepublik Deutschland, in: Czada, R./Wollmann, H. (Hrsg.): Von der Bonner zur Berliner Republik. 10 Jahre Deutsche Einheit, Leviathan, Sonderheft 19, S. 631-650
Grosser, D. (2003): Treuhandanstalt, in: Andersen, U./Woyke, W. (Hrsg.): Handwörterbuch des politischen Systems der Bundesrepublik Deutschland, Opladen, S. 626-630

Hauschild, P./Bischoff, J./Crummenerl, J./Gotthard, C./Harker, J./

Jekutsch, S./Pyka, C./Rühl, W. (2004): Privatisierung: Wahn \& Wirklichkeit. Kommunen im Fadenkreuz, Hamburg

Huffschmid, J. (2007): Die neoliberale Deformation Europas. Zum 50.

Jahrestag der Verträge von Rom, in: Blätter für deutsche und internationale Politik 3, S. 307-319

Janetschek, M. (2007): Privatisierungen und ÖPP als Ausweg? Kommunalfinanzen unter Druck - Handlungsoptionen für die Kommunen, Frankfurt a. $M$.

Kamm, F. (2003): Untersuchung und Vergleich der Privatisierung öffentlicher Unternehmen als zentraler Bestandteil der Wirtschaftspolitik in den achtziger und neunziger Jahren in Deutschland und Frankreich, Frankfurt a. M. u.a.

Lippert, I. (2005): Öffentliche Dienstleistungen unter EU-Einfluss. Liberalisierung - Privatisierung - Restrukturierung - Regulierung, Berlin

Mayer, F. (2006): Vom Niedergang des unternehmerisch tätigen Staates. Privatisierungspolitik in Großbritannien, Frankreich, Italien und Deutschland, Wiesbaden

Mittendorf, V. (2008): Bürgerbegehren und Volksentscheide gegen Privatisierungen und die Rolle der Gewerkschaften, in: Brand, T./Schulten, T./ Sterkel, G./Wiedemuth, J. (Hrsg.): Europa im Ausverkauf. Liberalisierung und Privatisierung öffentlicher Dienstleistungen und ihre Folgen für die Tarifpolitik, Hamburg, S. 310-329

Püttner, G. (2000): Daseinsvorsorge und service public im Vergleich, in: Cox, H. (Hrsg.): Daseinsvorsorge und öffentliche Dienstleistungen in der Europäischen Union, Baden-Baden, S. 45-55

Rügemer, W. (2006): Privatisierung in Deutschland. Eine Bilanz, Münster Ryner, M. (2003): Disciplinary Neoliberalism, Regionalization, and the Social Market in German Restructuring, in: Cafruny, A./Ryner, M. (Hrsg.): A Ruined Fortress? Neoliberal Hegemony and Transformation in Europe, Lanham u.a., S. 201-227

Sack, D. (2006): Liberalisierung und Privatisierungen in den Kommunen Steuerungsanforderungen und Folgen für Entscheidungsprozesse, in: Deutsche Zeitschrift für Kommunalwissenschaften 2, S. 25-38 Schröter, H. G. (2007): When Ugly Ducklings Grow Up: Cases from German Utilities in Energy, Transport and Telecommunications, in: Clifton, J./ Comín, F./Díaz-Fuentes, D. (Hrsg.): Transforming Public Enterprise in Europe and North America, Houndmills u.a., S. 77-89

Simonis, G. (Hrsg.) (1998): Deutschland nach der Wende. Neue Politikstrukturen, Opladen

Tofaute, H. (1994): Der große Ausverkauf. Die Privatisierung von Bundesunternehmen durch die Regierung Kohl, Köln

Zeuner, B. (1998): Das Politische wird immer privater. Zu neoliberaler Privatisierung und linker Hilflosigkeit, in: Heinrich, M./Messner, D. (Hrsg.): Globalisierung und Perspektiven linker Politik, Münster, S. 284-300 Zohlnhöfer, R. (2001): Die Wirtschaftspolitik der Ära Kohl, Opladen 